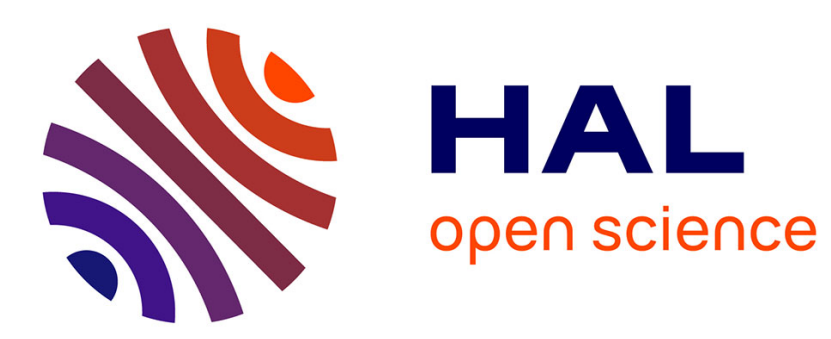

\title{
Vivre au rythme de la mer. Femmes de marins au travail sur les côtes nord de la Bretagne au XVIIIe siècle
}

\author{
Emmanuelle Charpentier
}

\section{To cite this version:}

Emmanuelle Charpentier. Vivre au rythme de la mer. Femmes de marins au travail sur les côtes nord de la Bretagne au XVIIIe siècle. Boudjaaba Fabrice. Le travail et la famille en milieu rural XVIe-XXIe siècle, PUR, pp.53-68, 2014, 978-2-7535-2910-6. halshs-01717127

\section{HAL Id: halshs-01717127 \\ https://shs.hal.science/halshs-01717127}

Submitted on 3 Apr 2018

HAL is a multi-disciplinary open access archive for the deposit and dissemination of scientific research documents, whether they are published or not. The documents may come from teaching and research institutions in France or abroad, or from public or private research centers.
L'archive ouverte pluridisciplinaire HAL, est destinée au dépôt et à la diffusion de documents scientifiques de niveau recherche, publiés ou non, émanant des établissements d'enseignement et de recherche français ou étrangers, des laboratoires publics ou privés. 


\section{"Uiure au rythme de la mer : femmes de marins au travail sur les côtes nord de la Bretagne au XUIII siècle» \\ dans BOUDJAABA, Fabrice, Le travail et la famille en milieu rural XUle-XX/e siècle, Rennes, PUR, 2014, P. 53-68 Emmanuelle CHARPENTIER'}

Résumé : Être une femme de marin, au XVIII ${ }^{\mathrm{e}}$ siècle, implique une dichotomie dans l'organisation de la vie familiale et du travail : d'une part, une vie de couple «normale » entre deux embarquements, et de l'autre, le temps de la séparation imposé par les «voyages sur mer» avec cependant, une perspective, celle du retour définitif à terre, à un moment ou à un autre. En effet, la plupart des marins des côtes nord de la Bretagne conservent un fort ancrage terrien et se sont souvent embarqués, faute de mieux à terre. Ces absences récurrentes, d'une durée variable selon les activités maritimes, obligent les ménages de gens de mer à s'adapter en optant pour l'endettement ou pour la pluriactivité, qui relève de l'évidence chez les «marins-ménagers». Les relations de pouvoir dans les ménages en sont perturbées en faveur des épouses dont l'autonomie se trouve renforcée pour certaines par la gestion active des biens de la communauté ou bien par une association avec d'autres femmes dans le cadre du travail ou de la cohabitation.

Mots clés : femmes, marins, gens de mer, absence, travail, associations féminines, autonomie

Abstract: To be a sailor's woman, in the XVIIIth century, involves a dichotomy in the organization of family life and work: a "normal" life with the husband, and the time of the separation because of the sea, with however, one perspective, the definitive return on the ground. Indeed, most of the sailors of the north coasts of Brittany have chosen the sea for lack of anything better on the ground. Because of these frequent absences, the sailors' households have to adapt themselves: some gets into debt, others chose the "pluriactivite ». The relations of power in the households are disrupted, in favour of the wives whose autonomy is strengthened for some by the active management of the properties' household or through the association with other women.

Keywords : women, sailors, absence, work, female associations, spinster clustering, autonomy

1 Docteur en histoire moderne, CERHIO UMR 6258, Université Rennes 2. 
« J'estimerés bien mieux que vous seriez chés nous. Je n'orés pas tant d'inquiétude comme j'ay et auray jusqu'à vostre retour, mais j'espère que, sy le Seigneur vous donne la grâce de revenir, que c'es le premier et le dernier voiage. ». Ces phrases émouvantes étaient adressées par MarieJacquette Pignot à son époux, parti de Saint-Malo le 18 novembre 1745 à bord du Condé, à destination de l'Amérique via $\mathrm{Cadix}^{2}$. Elles expriment la douleur de la séparation mais aussi un certain désarroi provoqué par l'absence : il s'agissait du premier voyage en mer de Gilles Pignot, un charpentier âgé de 38 ans. Ces quelques mots traduisent la difficulté à devenir femme de marin au $\mathrm{XVIII}^{\mathrm{e}}$ siècle et au-delà, enjoignent à dépasser les stéréotypes véhiculés par les marins eux-mêmes. Dans l'idéal, la femme de marin est considérée à travers son conjoint auquel elle doit l'abnégation la plus totale. Cette «identité par délégation ${ }^{3}$ » induit une hiérarchie des activités au sein du ménage, l'activité du conjoint étant largement mise en valeur en raison du parfum d'ailleurs qu'elle dégage, au détriment de celle de la femme qui reste de facto à terre. La difficulté consiste à saisir ces femmes de marins en tant qu'individus à part entière dans une société où la femme demeure une mineure du point de vue juridique, soumise à l'autorité de son époux. Or, l'absence, inhérente aux activités maritimes, bouleverse cet état de fait en imposant des temps de séparations durant lesquels les femmes se retrouvent seules et obligées de se débrouiller, intercalés avec des périodes de vie de couple «normale » entre deux embarquements. C'est là toute la spécificité des gens de mer et cela amène deux interrogations : quelles stratégies les ménages de gens de mer mettent-ils en œuvre afin de s'adapter aux rythmes de l'activité maritime ? Les rapports de pouvoir au sein du couple s'en trouvent-t-ils affectés ? Les paroisses du littoral nord de la Bretagne se prêtent bien à cette étude puisqu'elles comportent de nombreux ménages de marins au XVIII ${ }^{\mathrm{e}}$ siècle, entraperçus au détour des archives judiciaires et notariées, en complément des informations fournies par les Matricules des Classes. L'ensemble de ces documents permet d'appréhender l'absence en tant qu'élément constitutif de l'identité des gens de mer, d'analyser l'organisation du travail dans le ménage et enfin d'en mesurer l'impact sur les rapports de pouvoir au sein du couple.

\section{L'absence des hommes : un élément constitutif des ménages de gens de mer}

\section{A La mesure de l'absence}

Si l'absence est indubitablement liée au métier de marin, elle varie en fonction des activités maritimes, entre deux extrêmes. D'un côté, le long cours, qui implique des séparations d'au moins une année pour un voyage vers l'Amérique et jusqu'à un an et demi voire plus, pour un périple aux Indes ou en Extrême-Orient. De l'autre, des marins qui rentrent chez eux chaque soir, ceux engagés

2 Il s'agit de neuf lettres écrites entre le 29 novembre 1745 et le 2 octobre 1746, retranscrites dans HENwood P., « Marie-Jacquette Pignot : une femme de marin à Saint-Malo au XVIII ${ }^{\mathrm{e}}$ siècle », Mémoires de la Société d'Histoire et d'Archéologie de Bretagne, t. LXXVI, 1998, p. 321-339; lettre du 2 octobre 1746.

3 Voir les travaux de la sociologue Y. GUICHARD-CLAUDIC sur les pêcheurs thoniers de Concarneau : Éloignement conjugal et construction identitaire : le cas des femmes de marins, Paris-Montréal, L'Harmattan, 1999, 270 p. 
dans une navigation de proximité et les pêcheurs navigant à vue des côtes. Entre les deux se situent les marins qui pratiquent la grande-pêche, le cabotage ou la pêche hauturière : leur absence se compte selon les cas en semaines ou en mois. Ce sont ces derniers ainsi que les navigateurs au long cours qui ont retenu notre attention : leurs absences répétées ont un impact significatif sur leur vie de couple quand ils sont mariés. Le moindre imprévu, maladie ou accident, perturbe ces périodes de séparation d'une durée déjà incertaine. De même, la guerre prolonge l'absence : le service du roi oblige à s'embarquer plusieurs mois consécutifs et durant les conflits, fait courir le risque d'être blessé ou capturé d'autant que les Anglais n'hésitent pas à pratiquer une guerre «carcérale » qui atteint son apogée durant la Guerre de Sept ans (1756-1763).

Dans l'hypothèse où un réel attachement lie un marin à sa femme et à ses enfants, ces séparations récurrentes s'avèrent douloureuses. Le chagrin de Marie-Jacquette y est d'autant plus perceptible qu'elle se retrouve seule avec ses deux fils, pour la première fois : « Je ne puis vous marquer, mon cher mary, le chacrin où je suis de vostre départ. Il ne ce passe aucun moment que je ne pence en vous $^{4} »$. Au fil des lettres, écrites une année durant, la résignation finit par succéder à la peine : « Je vous asure, mon cher mary, que vostre absence m'ès toujous for sensible et bien ennuyeuse. Je voudrès que le voiage fut fait, mais je voye qu'il n'ès pas encore commencé. C'ès ce qui cause ma plus grande painne ${ }^{5} »$. Toutes comportent une prière afin que Gilles revienne sain et sauf. Cette peur émaille le quotidien de ces femmes de marins car maintenir un lien et avoir régulièrement des nouvelles est bien compliqué. Tant que le navire fait relâche dans une place portuaire française ou européenne, l'échange de nouvelles reste envisageable, à l'écrit ou de vive voix en utilisant un tiers revenant au pays, même si cela se réduit à des monologues informant en priorité l'autre de son état de santé. Marie-Jacquette s'y emploie puis narre à son mari ses soucis du quotidien et ses petites joies. La communication devient plus difficile après le départ vers un autre continent : seules les lettres déjà reçues maintiennent un lien artificiel mais concret avec ses proches ${ }^{6}$. Les rencontres fortuites permettent alors de faire passer un message sans savoir s'il arrivera à bon port, ni quand. C'est de cette manière que Jacques Gourdel, marin absent depuis 8 ans en 1709, fait parvenir des nouvelles à sa femme avec l'aide de deux navigants croisés par hasard et à quelques années d'intervalle, dans deux endroits très éloignés l'un de l'autre, Marseille et Carthagène ${ }^{7}$. Néanmoins, la plupart des femmes de marins demeurent sans aucune nouvelle récente : il leur faut vivre avec

4 HenWOOD P., op. cit., lettre du 29 novembre 1745.

5 Ibid., lettre du ler août 1746.

6 C'est peut-être la raison pour laquelle Gilles Pignot avait gardé celles de sa femme dans ses affaires.

7 Arch. Dép. d'Ille-et-Vilaine [ADIV], Juridiction de Saint-Ideuc, 4B3341, attestation du 30 avril 1709. Voir CHARPENTIER E. «Incertitude et stratégies de survie : le quotidien des « femmes de partis en voyage sur mer » des côtes nord de la Bretagne au XVIII ${ }^{\mathrm{e}}$ siècle ", dans PLOUX F. (dir), Usages et représentations du temps dans les sociétés littorales, Annales de Bretagne et des Pays de l'Ouest, t. 117, n³, septembre 2010, p. 39-54. 
l'incertitude et l'éventualité de la mort.

Cette incertitude permanente quant au sort du conjoint pousse quelques femmes à rompre leur solitude et à se remarier, la mort étant présumée. De ce fait, un retour tardif réserve parfois des surprises : après 19 ans passés « au service de sa majesté » sans avoir donné la moindre nouvelle, Hervé Kergil découvre que sa femme s'est remariée ${ }^{8}$. Huit jours après le mariage, l'ancien époux vient réclamer sa femme « comme l'ayant aussi épousée en face d'église depuis environ vingt-trois ans » ce qui provoque l'annulation du second mariage. Ces quelques moments passés ensemble, des années auparavant, ont effacé 19 années d'absence d'où ces retrouvailles heureuses bien qu'un peu mouvementées.

\section{B La perspective du retour définitif à terre}

Les vieux marins se font rares à bord des navires ce qu'indiquent les registres établis par l'administration des Classes. La plupart des marins sont des hommes jeunes qui naviguent depuis l'adolescence, voire l'enfance ${ }^{9}$. Une telle disproportion dans les effectifs suggère soit des décès prématurés, soit des interruptions, volontaires ou non, de la carrière maritime : pour prendre le large, mieux vaut être en forme et en bonne santé car à bord, l'organisme est mis à rude épreuve. La surmortalité maritime est une réalité dont les marins sont bien conscients ; ils savent également que la mer peut blesser gravement et contraindre à un arrêt anticipé et définitif tout en laissant des stigmates sur le corps. Les registres tenus par les Commissaires aux Classes regorgent d'exemples d'hommes encore jeunes devenus des « hors de service » à l'image de ce matelot de Pléneuf, déclaré comme tel après être tombé de la grande vergue et s'être « fracassé la tête et cassé les cuisses ${ }^{10}$ ». Naviguer présente donc des risques et correspond à une période de la vie bien précise, la jeunesse, en gardant à l'esprit que la situation reste précaire et peut s'arrêter du jour au lendemain. D'autant que pour de très nombreux marins, le choix de la navigation ne constituait pas la réponse à un irrésistible « appel du large ». Même chez les plus favorisés, le basculement dans la vie maritime répond à des ressorts complexes : un passage obligé dans un cursus de formation destiné aux futurs négociants ou une tradition chez les membres de l'état-major issus de la noblesse. Pour les autres, la familiarisation précoce avec l'horizon maritime semble être déterminante quand la mer est partie prenante de l'espace familier, celui du quotidien : c'est le cas, par exemple, des « enfants du port ${ }^{11} »$, de Plérin, qui traînent sur les quais. Côtoyer des gens de mer peut encourager à prendre le large.

8 Arch. Dép. du Finistère [ADF], 4E110 14, requête du 5 octobre 1752.

9 Un sondage mené dans les rôles de 1751à 1762 dans les quartiers de Saint-Brieuc et de Morlaix et Roscoff montre un âge moyen de 15 ans pour un total de 249 mousses. Service Historique de la Marine [SHM, Brest], quartier de Saint-Brieuc, 4P3 13, registre des mousses 1751-1762 et quartier de Roscoff et Morlaix, 6P3 19, registre des mousses 1751-1762.

10 Ibid., quartier de Saint-Brieuc, 4P3 13, registre des mousses, 1751-1762, Pléneuf.

11 Arch. Dép. des Côtes d'Armor [ADCA], B3756, information du 3 janvier 1785. 
Gilles Pignot était peut-être dans ce cas : sa vocation très tardive pourrait s'expliquer par l'influence de son frère cadet, tonnelier-navigant depuis l'âge de 16 ans $^{12}$. La filiation professionnelle joue aussi certainement un rôle car l'apprentissage de la mer se transmet tout autant par le geste. Certains enfants ont déjà navigué avant leur inscription officielle sur les registres des Classes, comme Jacques Marie Coet, de Plounéour-Trez, navigant depuis l'âge de 5 ans et dont le père était maître de barque $^{13}$. Un sondage réalisé sur 532 mousses de 14 paroisses des quartiers de Saint-Brieuc, Morlaix et Roscoff confirme en partie cette hérédité professionnelle : $62 \%$ d'entre eux ont un père navigant ${ }^{14}$ mais cela signifie aussi que les $38 \%$ restants sont fils de terriens. La raison réside dans le manque de perspectives à terre. S'embarquer s'apparente à la saisie d'une opportunité, faute de mieux. Une situation familiale difficile, la perte des parents, la pauvreté poussent à saisir les occasions de travail qui se présentent et s'embarquer en est bien une, sur le littoral. Le rapport établi le 15 juin 1769 par le capitaine de La Rosalie, navire armé à Saint-Malo, est éloquent ${ }^{15}$ : il y déclare la présence d'un passager clandestin, Pierre Richard, orphelin âgé de 19 ans. Caché à bord, il n'a révélé sa présence qu'une fois en mer. Interrogé par le capitaine, il lui répond « que c'était l'envie de travailler et de naviguer et de plus de trouver son pain assuré pendant le voyage et peut-être quelques hardes vu qu'à terre il était sans hardes, et à la requête d'un parent à lui demeurant dans le bourg de Paramé, qu'il avait travaillé pendant l'armement du navire au métier de dos blanc [le transport du lest] ce qui lui avait donné encore plus d'ardeur de naviguer et qu'ayant gagné quelques journées il avait acheté quelques hardes dans le dessein de se cacher à bord et faire le voyage ». Pour les terriens « dépendants » vivant sur le littoral, prendre la mer apparaît comme une alternative économique non négligeable fournissant un travail et un salaire. Cela permet de contourner un rapport à la terre très valorisé, en l'absence de capital financier ou foncier. L'exemple des deux plus jeunes fils de Jean Ledaguenel et sa femme, laboureurs à Langrolay, est significatif : ils sont dans le « dessein l'un et l'autre de prendre le party et l'art de s'embarquer pour la navigation ${ }^{16}$ », quand leurs parents prennent conscience de leur incapacité à faire valoir seuls leur métairie sans leurs deux fils. Ils leur proposent donc de rester, moyennant une pension de 50 livres versée à chacun d'eux. Ici, la vocation maritime est subordonnée au rapport à la terre.

D'ailleurs, la finalité d'une carrière maritime demeure pour beaucoup l'horizon terrestre ; les registres des Classes donnent des exemples de marins qui, après une carrière honorable « renoncent

12 HENWOOD P., op. cit.

13 SHM, quartier de Roscoff, 6P3 20, registre des matelots et des officiers mariniers, 1776-1787, Plounéour-Trez.

14 Ibid., quartier de Saint-Brieuc, 4P3 13, registre des mousses 1751-1762 et quartier de Roscoff et Morlaix, 6P3 19, registre des mousses 1751-1762

15 ADIV, 9B327, rapport du capitaine de La Rosalie, 15 juin 1769.

16 Les autres enfants s'étant mariés, et ledit Ledaguenel ayant « donné leur ménage et fait de la dépense pour les établir »; ibid., 4E11552, déclaration du 24 mai 1751. 
à la mer » pour s'installer définitivement à terre et exercer une autre profession. La navigation a servi à accumuler un pécule laissant espérer une vie moins dangereuse à terre. Après 12 années de pêche suivies de 4 ans au service du roi, Vincent Jolivet, de Plouescat, devient fermier, la trentaine passée $^{17}$. Certains reprennent la profession de leur père : François Tanguy, fils d'un fermier de Cléder, effectue plusieurs campagnes au service du roi puis devient tardivement laboureur, à 35 ans $^{18}$. La reconversion peut être facilitée par des investissements, considérés comme un moyen d'anticiper la fin de la carrière maritime. Le mode de versement des salaires facilite les acquisitions : versés ponctuellement, ils permettent de disposer de sommes assez élevées et en liquidités. Les biens fonciers dominent dans les achats effectués par les gens de mer. La stratégie suivie est à court terme de s'assurer des revenus complémentaires, sécurisants face à la précarité des carrières maritimes. Soit l'acquéreur met lui-même en valeur son bien, soit il le fait exploiter contre le versement d'une rente annuelle afin à long terme, d'assurer ses vieux jours et transmettre un héritage à ses enfants. En cela, les gens de mer se conforment aux stratégies menées par les terriens, en fonction du cycle de vie ${ }^{19}$. Les opérations passées devant notaire pourraient correspondre à sa seconde phase durant laquelle un individu dispose éventuellement d'héritages, d'une faculté d'emprunt plus importante et surtout de liquidités liées ici à l'expérience maritime acquise au fil des voyages, induisant un salaire plus élevé. Aussi, chacun achète à la mesure de ses moyens mais cela rend possible l'accès à la propriété pour un matelot, qu'il s'associe avec d'autres acheteurs ou achète seul, tel Julien Eon, un « garçon », marinier calfat de Pleurtuit, qui devient propriétaire en l'espace de 3 semaines de trois pièces de terre et un « aplacement de maison et étable » pour un total de 1078 livres payées comptant ${ }^{20}$. Pour l'état-major, il s'agit davantage de consolider le patrimoine initial en saisissant les opportunités d'achat qui se présentent : Claude Dominique Avice, capitaine noble de vaisseau de Cancale, pratiquant la grande-pêche et la course, accumule les possessions de 1766 à 1783, année de sa mort. Durant ces 17 ans, il a mis en ferme trois métairies et a acquis quatre pièces de terre dans la paroisse de Cancale, pour un total de 1066 livres $^{21}$. Il adopte une stratégie d'accumulation qui consiste à acheter des biens pour les mettre en valeur en faire-valoir-indirect.

17 SHM, quartier de Roscoff et de Morlaix, registre des officiers mariniers et des matelots, 6P3 20, 1776-1787, Plouescat.

18 Ibid., Cléder.

19 Voir à ce propos BOUDJAABA, F., Des paysans attachés à la terre? Familles, marchés et patrimoines dans la région de Vernon (1750-1830), Paris, PUPS, p. 149-158, VIGNERON S., « La sphère des relations foncières des ruraux : l'exemple du Cambrésis (1681-1791) », Histoire et Sociétés rurales, n 20, 2003, p. 53-77 et BEAUR G., «Investissement foncier, épargne et cycle de vie dans le pays chartrain au XVIII ${ }^{\mathrm{e}}$ siècle », Histoire et mesure, vol. 6, n³/4, 1991, p. 275-28 et «Le marché foncier. Conjoncture des transferts de propriété et stratégies d'accumulation (France, XVIII ${ }^{e}$ siècle) », dans BEAUR G. et alii, Les sociétés rurales en Allemagne et en France (XVIII et XIX siècle), Rennes, Association d'Histoire des Sociétés Rurales, 2004, p. 213-229

20 ADIV, 4E11551, contrats de vente des 22 et 28 juin, $1^{\text {er }}$ et 15 juillet 1743.

21 Ibid.,4E1507, contrat de vente du 19 juillet 1766, bail à ferme du 22 novembre 1766, contrat de vente du 23 décembre $1771 ; 4 \mathrm{E} 4692$, contrats de vente du 15 février 1772 et du 5 février $1773 ; 4 \mathrm{E} 4695$, baux à ferme du 29 mars 1781 et du 24 mai 1783 . 
Claude Dominique Avice ne fait qu'agrandir son patrimoine ce que les revenus issus de la course ont dû largement faciliter. En effet, certaines activités sont plus lucratives que d'autres : les choix effectués en matière d'embarquement influent sur les possibilités d'accès au marché foncier, de même qu'une ascension rapide dans la hiérarchie du bord.

\section{Une adaptation forcée des ménages aux rythmes de la mer}

\section{A Endettement et pluriactivité}

La discontinuité des revenus maritimes affecte profondément les ménages de gens de mer : il faut apprendre à ne pas compter dessus. C'est ce que découvre Marie-Jacquette : dans ses lettres, elle ne cesse de demander de l'argent à son mari au début, timidement, puis elle s'enhardit lorsqu'elle apprend le versement de quelques mois de salaire. Elle lui reproche presque son départ dans une lettre écrite probablement sous le coup de la colère : «Voilà ce que c'és que l'oingnement [l'éloignement]. Je voye que vous m'avés laiscé dans un grand embara, chargé de deux enfan et une louage [loyer] de cinquante écus ${ }^{22} \gg$. Finalement, Gilles lui fait passer 10 écus juste avant de quitter Cadix. Entretemps, elle a dû se débrouiller seule : elle évoque à plusieurs reprises la mise en gage de leur «argenterie » et la vente de ses hardes. Elle enjoint aussi Gilles de jouer de ses relations pour qu'elle puisse emprunter de l'argent. A l'instar d'autres femmes de marins, il faut subvenir aux dépenses courantes ce que montrent les listes de dettes figurant dans les actes rédigés lors du décès d'un des conjoints. Sur 275 documents analysés, 84 mentionnent explicitement des créances, soit $30,5 \%$ des $\operatorname{cas}^{23}$. Pour presque la moitié, l'endettement correspond à moins de $25 \%$ de la valeur de l'inventaire lors de la rédaction de l'acte ce qui ne remet pas vraiment en cause l'équilibre du ménage. Les dettes couvrent largement les dépenses du quotidien à côté de celles, plus ponctuelles, liées à un événement familial, à des investissements et à des prêts d'argent. Sans être généralisé, l'endettement est une pratique assez répandue parmi les gens de mer, des matelots jusqu'à l'étatmajor, l'absence étant considérée par les créanciers comme une circonstance atténuante.

Une carrière maritime suit une trajectoire en pointillés qui correspondent aux embarquements successifs. Entre ces derniers s'écoulent des périodes plus ou moins longues, en attente d'un nouveau départ. C'est durant celles-ci qu'une majorité de gens de mer pratique une autre activité concomitante, rendue nécessaire pour assurer la continuité de leurs revenus, aussi modiques soientils. Les salaires, précédés d'avances, sont en général versés au retour, en liquidités, ce qui signifie parfois des sommes importantes correspondant à une campagne de pêche, ou à plusieurs semaines, mois, voire années de travail à bord. Le montant exact est rarement connu à l'avance et peut être versé tardivement. Seuls les plus aisés peuvent donc se permettre de rester longtemps à terre sans

22 HeNWOOD P., op. cit., lettre du 20 juin 1746.

23 Ce chiffre est certainement sous-évalué en raison des dettes contractées oralement. 
travailler. Les autres, pluriactifs, apparaissent subrepticement au détour des sources qui révèlent des combinaisons tournées vers les activités maritimes, paramaritimes et terriennes. Dans celles-ci, la hiérarchie du bord se superpose à la la hiérarchie à terre : les matelots exercent en général des activités dans l'artisanat, le petit commerce ou l'agriculture. Au mieux, ils sont meuniers ou laboureurs et au pire, ils se retrouvent journaliers ou batteurs en grange alors que les capitaines de navire penchent du côté de la bourgeoisie urbaine et marchande.

La pluriactivité prend toute son ampleur quand elle est pratiquée à l'échelle du couple. MarieJacquette mentionne dans ses lettres son «petit commerce» de denrées, installé dans une «boutique » à Saint-Malo ${ }^{24}$. Elle fait partie des « regratières » malouines, pour beaucoup femmes de matelots ou d'officiers mariniers ${ }^{25}$. D'autres se font cabaretières ou aubergistes, boulangères, fournières par exemple, ou exercent une activité en lien avec le textile. Des professions découlent directement de l'activité portuaire telles les «couturières en voile» ou les «cordelières journalières ». Cela ne permet guère de dépasser le « seuil de l'indépendance» : pour celles qui travaillent pour survivre, la pluriactivité est une nécessité au-delà de la revendication d'un métier à soi, faisant part égale avec l'activité du conjoint. Cette forme de reconnaissance sociale semble moindre à la campagne : peu de femmes mentionnent une profession, excepté quelques « ménagères » dans les paroisses de la côte de Goëlo et à Trévou-Tréguignec. La transposition du terme « ménager » au féminin pourrait signifier une reconnaissance du travail agricole effectué par la femme mais aussi une forme de prise de conscience de son rôle économique dans le couple, dans les paroisses rurales et littorales. Toutes les femmes de marins nécessiteuses n'exercent pas forcément un métier précis mais cela ne signifie pas pour autant qu'elles ne travaillent pas : nombre d'entre elles exercent des tâches ponctuelles et peu qualifiées, rémunérées à la journée afin d'améliorer l'ordinaire et nourrir leurs enfants. Dans les paroisses rurales du littoral, les possibilités d'emploi sont nombreuses mais saisonnières, comme le montre une affaire d'infanticide commis en 1761 par la femme d'un soldat, à Cancale ${ }^{26}$. Au cours de ses vacations, elle côtoie d'autres femmes, toutes des filles, épouses ou veuves de marins, qu'elle ramasse des pommes, du goémon ou du bled noir, batte du froment, « scie » du bled noir, « fasse du bled » ou participe à la « façon du froment ». Ces femmes liées au milieu maritime, dans le besoin et sans ressources propres, constituent une main d'œuvre d'appoint pour ces tâches agricoles saisonnières. Sur le littoral s'y ajoutent les travaux liés à l'exploitation de l'estran : une autre affaire met en scène six femmes ou veuves de matelots

24 HenWOOD P., op. cit., lettre du 24 janvier 1746.

25 D'après LeSPAGNOL A., «Femmes négociantes sous Louis XIV : les conditions complexes d'une promotion provisoire », dans CROIX A., LAGREE M. et QueniarT J. (dir.), Populations et cultures, études réunies en l'honneur de François Lebrun, Rennes, 1989, Amis de François Lebrun, p. 463-470.

26 ADIV, 4B1057, plainte du $1^{\text {er }}$ avril 1761. 
gagées à la journée pour ramasser et parquer les huîtres à Cancale ${ }^{27}$. Dans les places portuaires, les opportunités de travail sont sans doute plus larges mais la tentation de l'illicite se fait plus bien plus grande $^{28}$, en servant par exemple de «petites mains » pour le trafic de « faux-tabac ». En outre, les femmes dans le besoin, vivant près de centres urbains tels Saint-Malo, bénéficient d'opportunités de travail supplémentaires : Marie Gaubert, épouse de matelot, et sa sœur accueillent deux nourrissons qu'elles ont « à nourrir », en échange de 40 livres par an, en plus du salaire du mari, marin au long $\operatorname{cours}^{29}$.

\section{B Le cas des « marins- ménagers » et de leur femme}

Si ces «marins-ménagers » se déclarent rarement comme tels, ils sont toutefois repérables dans les baux à moitié ou à ferme. Parmi les biens pris en location, une partie concerne des exploitations agricoles, par exemple, le bail de huit ans conclu par Jean Hamelin, un navigant de Cancale et sa femme en 1770 ; il comprend une maison, une étable, un cellier et un jardin, deux pièces de terre, un petit verger, un clos et des jannais ${ }^{30}$. Il est donc possible d'exercer de façon simultanée activités maritime et agricole pour diversifier les sources de revenus. Un éclairage complémentaire est apporté par les actes décrivant les biens d'un individu à un moment de sa vie, en particulier les inventaires après décès. Ils permettent de repérer des marqueurs attestant de pratiques pluriactives, pour l'agriculture, la possession d'une charrue, d'outils agricoles, de réserves de semences, d'animaux, de levées de terre et pour le textile, des outils spécifiques ou des stocks de fil ou de la filasse, constituent autant d'indices. D'après ces critères, les trois-quarts des actes analysés ${ }^{31}$ font état de pratiques pluriactives tournées de préférence vers le textile puis vers l'agriculture. Pour une majorité, et tout le long des côtes nord de la Bretagne, les deux activités sont associées en raison de leur caractère complémentaire. Par exemple, Michel Leostic, maître de barque de Porspoder, possède en 1780 un cochon, une vache et une génisse, des outils agricoles, plusieurs mulons à battre ou déjà battus, de l'avoine, des pois roux, des choux et des panais, un mulon de fumier avec du goémon, 200 poignées de lin broyé, deux poids de lin peigné, un dévidoir à fil cru et une « pecelle à peceler le $\operatorname{lin}^{32} »$. Cette pluriactivité est le fait de presque tous les gens de mer, des membres de l'état-major aux matelots en passant par les pêcheurs. Les différences s'expriment en termes de degré d'investissement personnel et familial, perceptible à travers le nombre de marqueurs, leur rareté et leur degré de perfectionnement. Tout dépend de la place accordée par le ménage aux

27 Ibid.,9B319, interrogatoires du 9 août 1766.

28 Voir VARY M., « Les multiples facettes de l'économie parallèle dans les villes maritimes au XVIII ${ }^{\mathrm{e}}$ siècle » dans GARNOT B. (dir.), Justice et argent. Les crimes et les peines pécuniaires du XIII au XXI siècle, Dijon, EUD, 2005, p. 77-85.

29 ADIV, 4B1058, plainte du 14 février 1765.

30 Ibid., 4E1509, bail à ferme du 10 avril 1770 .

31 Sur 275 documents analysés.

32 ADF, B2575, inventaire de communauté du 19 août 1780. 
activités terriennes : un simple appoint, un complément non négligeable ou une activité à part égale. Ces actes suggèrent un fort ancrage rural des populations navigantes et posent la question de la continuité des activités terriennes lors de l'absence du conjoint. Si celui-ci opte pour la pêche, son caractère saisonnier permet de s'organiser selon un calendrier annuel défini à l'avance. Dans le cas de la grande pêche, pratiquée dans le pays malouin et aux environs de Binic, les départs se font en avril pour un retour prévu en octobre, le reste de l'année étant consacré au travail agricole. La combinaison entre les différentes activités est plus poussée pour les pêcheurs de maquereau : comme la pêche se fait d'avril à juin, ils se consacrent aux travaux agricoles le reste du temps et dans les périodes creuses, au ramassage du goémon et du sable marin.

L'absence ne semble pas rédhibitoire pour les propriétaires car ils n'ajoutent pas de clauses spécifiques dans les baux conclus avec les gens de mer. La description des biens et les charges imposées aux preneurs laissent souvent à penser qu'une présence constante est requise sur place. Cela n'empêche pas Louis Poidevin, un navigant, et sa femme, de louer la métairie de la Brouesnière, à Cancale, depuis 1764 , avec succès puisque la ferme est reconduite ${ }^{33}$. Le pari est plus poussé pour les afféagements puisqu'ils reposent sur l'obligation d'améliorer un terrain « vague » en échange d'une rente modique versée chaque année. L'un concerne un marinier de Cancale et sa femme, afféagistes d' un journal de terre alors qu'ils possèdent déjà une pièce de terre ${ }^{34}$. Toutefois, pour une exploitation, petite ou grande, il faut assurer le versement du louage chaque année. Dans ce cas, le travail effectué par l'épouse est essentiel : durant les périodes d'absence, elle assure seule les travaux agricoles, aidée parfois par un domestique, avec un éventuel recours à une main d'œuvre d'appoint. Encore faut-il en avoir les moyens : mettre à contribution sa famille, ses enfants, est sans doute une solution courante. Les femmes plus aisées font faire les tâches les plus difficiles : des mentions d'impayés figurent dans plusieurs listes de dettes. La veuve de Guillaume Leprince, tonnelier au service du roi, doit 15 livres à Jean Raffray « pour avoir manué et fait deux journaux de froment sur sa terre ${ }^{35} »$. Le bail de la métairie de la Villeneuve met en évidence une autre solution, plus originale ${ }^{36}$ : une association entre les preneurs, d'un côté Pierre Gaultier, navigant, et sa femme, Marie Guilbert, et de l'autre, Alain Guilbert, un laboureur. La similitude des noms laisse à penser qu'ils sont apparentés. Les absences de Pierre Gaultier sont palliées par la présence constante de sa femme et par celle de son probable beau-frère.

Dans les ménages de «marins-ménagers », la femme occupe donc une place prépondérante du fait de son ancrage à terre. L'absence induit par la force des choses une division sexuée du travail au

33 ADIV, 4E1509, bail à ferme du 22 février 1770.

34 Ibid., 4E1503, , afféagement du 30 août 1753.

35 Ibid., 4B1329, inventaire après décès du 18 juin 1781 et 4B3444, apposition de scellés du 17 février 1741.

36 A Cancale. Ibid., 4E1507, bail à ferme du 7 janvier 1766. 
sein du couple : l'homme se tourne vers l'extérieur, l'horizon maritime tandis que la femme assure l'activité complémentaire et génératrice de revenus plus réguliers, à terre. Cela rend d'autant plus visible le travail de ces femmes dans une société où les activités domestiques suscitent peu de reconnaissance sociale. Tout dépend de la durée de l'absence : pour les pêcheurs, cette répartition du travail reste temporaire. Pour les autres, l'absence est plus durable et se traduit par une féminisation des tâches traditionnellement dévolues aux hommes. Les épouses en viennent alors à assumer la charge de l'exploitation agricole ce qu'a aussi observé Dominique Guillemet dans les îles de l'Ouest $^{37}$. Il est alors difficile d'imaginer une redistribution des rôles lors du retour du conjoint, sous réserve qu'il revienne en bonne santé : les rapports de pouvoir au sein du ménage s'en trouvent certainement bouleversés, au profit des femmes.

\section{Vers un rééquilibrage des rapports de pouvoir au sein des couples?}

\section{A Une gestion active des biens de la communauté}

La gestion des biens de la communauté est officialisée par une procuration établie devant un notaire et à la demande du conjoint. Ce document officiel est valable uniquement le temps de l'absence et doit être renouvelé à chaque embarquement, souvent dans l'urgence du départ. L'épouse est la bénéficiaire toute désignée à laquelle sont conférées des compétences étendues. A titre d'exemple, voici la procuration de Joseph Lelionnais, un marin de Pleurtuit, « sur le point de s'embarquer pour le voyage de la côte de guines [Guinée] », en faveur de son épouse, datée de 1774 : «...faire pendant mon absence toutes les affaires que je pourais faire moy mesme sies j'etais sur les lieues, intanters toutes sorte de demande en justice de defandre contre celles quy nous sons sinifies [signifiées] ou quy pouraies lestre de poursuivres ou traiters surs le tout comme bon lui sembleras de vandre et dacheter et de doners des quittance approuvant sans reserve tout ce quel feras quans mesme cela ne seraie pas expliquer par la presante ${ }^{38} \gg$. Il lui accorde donc une pleine et entière confiance, un peu forcée, pour l'administration de ses affaires du couple ${ }^{39}$.

Un tel acte donne la possibilité aux femmes d'engager leur communauté devant notaire, en l'absence de leur conjoint. Sur l'ensemble des signataires ${ }^{40}$, plus d'un tiers des époux est absent, en mer, lors de l'officialisation des documents à l'étude : les femmes, en vertu de leur procuration, signent seules des actes importants sans que personne n'émette de réserves. Ainsi, Gillette Chevalier achète une

37 Guillemet D., Les îles de l'Ouest, de Bréhat à Oléron du Moyen Age à la Révolution, La Crèche, Geste Éditions, 2007,572 p.

38 ADIV, 4E11558, procuration du 20 février 1774.

39 Nicole DUFOURNAUD et Bernard MiCHON ont retrouvé à Nantes des procurations émanant de capitaines de navires s'apprêtant à partir pour un voyage au long cours : elles sont rédigées dans des termes semblables ; « Les femmes et le commerce maritime à Nantes (1660-1740) : un rôle largement méconnu », Clio, n 23, 2006, p. 311-330. Les migrations montagnardes obligent aussi les hommes disposant d'un peu de bien à signer des procurations élargies en faveur de leur épouse ; voir POITRINEAU A., Remues d'hommes. Les migrations montagnardes en France, 17e-18e siècles, Paris, Aubin, 1983, 325 p.

40 Pour un ensemble de 321 actes notariés. 
maison et ses dépendances à Ploubalay, son mari étant «sur les vaisseaux de la Compagnie des Indes $^{41} »$. Il arrive que la signature de l'acte se fasse uniquement entre femmes $\mathrm{d}^{\prime} \ll$ absents en voyage sur mer » : quand, en théorie, Louis Lhostellier, maître charpentier navigant de Pleurtuit, achète en 1746 une terre pour 250 livres à Joseph Jarnigon, maître calfat de Lorient, ce sont leurs épouses qui concluent l'acte car les deux hommes sont absents ${ }^{42}$. Les femmes apparaissent donc comme des contractantes à part entière, certes «autorisées » par leur mari mais qui profitent de cette autonomie temporaire. Toute la question est de savoir si elles suivent des directives données par leur conjoint avant son départ ou bien si elles agissent de leur propre chef. Les deux situations coexistent certainement.

Quelques documents laissent entrevoir des femmes prenant des initiatives avec la bénédiction de leur conjoint. François Perrigault félicite sa femme dans une lettre faisant office de procuration pour un achat déjà conclu verbalement sur son initiative à elle, mais non encore officialisé devant un notaire $^{43}$. La procuration figure d'ailleurs dans l'acte de vente de deux maisons pour un montant de 850 livres. Cela va beaucoup plus loin pour Claude Helvant, sieur de la Villegris, et son épouse, de Cancale : ce capitaine de vaisseaux marchands en fin de carrière, noble, mène dans les années 1770 une stratégie d'accumulation en multipliant les achats immobiliers et les prêts d'argent pour plus de 4000 livres. Or, la moitié des actes sont signés en son absence par Geneviève Baudouin, son épouse et partenaire en affaires. En 1771, elle conclut en mai une reconnaissance de dettes de 500 livres, une autre, le même jour, de 400 livres, et 2 mois après, l'achat d'une terre pour 330 livres. En 1772, elle achète une maison pour 300 livres, puis en 1773, deux pièces de terre pour 160 livres et 3 mois après, une maison pour 200 livres. En 1776, elle accorde un prêt de 100 livres. L'ensemble correspond à un montant total de 1990 livres $^{44}$ : la gestion d'une telle somme, même fractionnée, ne peut que reposer sur de solides liens de confiance établis entre époux et symbolisés par une donation mutuelle conclue en 1773 devant notaire ${ }^{45}$. Ce n'est pas sans rappeler la situation de ces « femmes négociantes » présentes au moins à Nantes, aux Sables d'Olonnes mais aussi sur les côtes

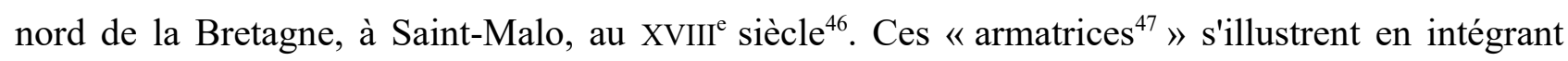
l'activité d'armement de navires d'ordinaire dévolue aux hommes car toutes ces femmes ont repris les affaires de leur défunt mari, armateur et négociant de profession. Comme le souligne André

41 ADIV, 4E11553, bail à ferme du 20 octobre 1758.

42 Ibid., 4E11552, contrat de vente du 16 juillet 1746.

43 ADCA, 3E34 11, lettre du 25 janvier 1780 et contrat de vente du 16 février 1780.

44 ADIV, 4E1510, reconnaissances de dettes du 1er mai 1770 et contrat de vente du 22 juillet 1771 ; 4E4692, contrats de vente des 2 janvier 1772, 6 juillet et 14 octobre 1773, reconnaissance de dettes du 11 juin 1776.

45 Ibid., 4E4692, donation mutuelle du 8 avril 1773.

46 Dufournaud N. et Michon B., op. cit., et des mêmes auteurs, « Les femmes et l'armement morutier : l'exemple des Sables d'Olonnes pendant la première moitié du XVIII ${ }^{\mathrm{e}}$ siècle », Annales de Bretagne et des Pays de l'Ouest, t. 110, $\mathrm{n}^{\circ}$ 1, 2003, p. 93-113; LESPAGNOL A., op. cit.

47 Dufournaud N. et Michon B., «Les femmes et l'armement... », op. cit., p. 99-100. 
Lespagnol, encore faut-il qu'elles disposent des compétences nécessaires pour exercer de telles fonctions : elles ont été mêlées du vivant de leur époux à la gestion de ses affaires en tant $q^{\prime} u^{\prime} «$ auxiliaire privilégiée ${ }^{48} »$. Par ailleurs, toutes ont évolué dès leur plus jeune âge dans un « fond de culture marchande » tourné vers la $\operatorname{mer}^{49}$. On ne peut s'empêcher d'esquisser un parallèle avec Geneviève Baudouin qui, sans être mariée à un négociant, est largement associée aux affaires de son époux.

\section{B Des associations de femmes}

Ces associations de femmes - que laissent entrevoir les archives - forment une réponse à l'univers très masculin du navire. Le plus souvent informelles, elles recouvrent des formes variées selon le degré d'investissement personnel de leurs membres.

Les plus élaborées sont les « sociétés pour l'amarinage et le commerce des huîtres » présentes à Saint-Malo. L'une d'elles est dirigée par Jeanne Fougeray, une marchande d'huîtres, femme de navigant, l'autre par une poissonnière, Marie Boistard, dont le mari est «absent en mer ${ }^{50} »$. La première regroupe les deux filles de Jeanne Fougeray, une autre femme, une veuve et une domestique tandis que la deuxième est composée de la sœur de Marie Boistard, d'une veuve et d'une fille majeure. Les deux « chefs » se sont mises d'accord pour que l'achat des huîtres se fasse selon des modalités précises. Mais il y a des ratés et dans ce cas, l'affrontement se règle sur la grève, société contre société : c'est précisément l'objet de la plainte déposée par Jeanne Fougeray le 27 février 1769. Accusée d'avoir acheté des huîtres sans respecter le règlement, elle se fait agresser dès le lendemain matin alors qu'elle est en train d'ouvrir ses huîtres avec ses « compagnes », en bas de la cale. Marie Boistard et ses «consortes», accompagnées de sa cousine et de quatre autres femmes, surgissent brusquement et renversent le cuveau d'huîtres « écallées » sur le sable, la rouent de coups et « vomissent plusieurs injures et invectives », encouragées de loin par la mère de Marie Boistard. Selon un témoin, «la dispute devint générale, et se frappèrent les unes et les autres réciproquement pendant environ un demi quart d'heure »; seule l'apparition d'un couteau met fin à l'altercation. Cette affaire montre que chaque «société» fonctionne comme un clan composé uniquement de femmes apparentées ou non. Les hommes en sont complètement absents : l'écaillage des huîtres est clairement une tâche sexuée dont ils sont exclus pour être cantonnés à la pêche. Une situation similaire se retrouve à Cancale où des « maîtresses de bateaux » ou « appareilleuses » s'occupent le plus souvent avec des journalières - sans organisation comparable aux sociétés malouines - du parcage et de la préparation des huîtres, pêchées par les hommes et destinées aux

48 LESPAGNOL A., op. cit., p. 468.

49 Ibid., p. 469.

50 ADIV, 9B324, février-mars 1769. 
navires étrangers venus en acheter ${ }^{51}$.

L'activité maritime des conjoints amène aussi à trouver des solutions pratiques pour pallier les effets de l'absence, en particulier pour le travail de la terre. Cela peut prendre la forme d'une association de gens de mer à l'image de celle formée pour la mise en valeur d"un terrain de six journaux et demi, à Cancale ${ }^{52}$. Les preneurs sont une veuve, parente a priori du sieur Louis Renard, autre afféagiste avec sa femme ainsi qu'un autre couple. Les deux hommes, lors de la conclusion de l'acte, sont « absents en voyage sur mer » et ce sont les femmes qui signent, dûment autorisées par eux. Le défi est d'ampleur mais ne paraît pas effrayer les trois femmes. L'initiative paraît relever dans ce cas de celles-ci, les époux ayant donné leur accord : on pressent qu'elles comptent bien s'occuper ellesmêmes de l'amélioration du terrain du fait de leur présence continue à terre et en dépit de l'aide ponctuelle de leur mari.

La cohabitation est peut-être la forme d'association la plus originale chez les gens de mer d'autant qu'elle est présente tout le long des côtes nord de la Bretagne : elle repose sur la présence dans un même logement d'une «famille conjugale » soit un couple et ses éventuels enfants, et d'un ou de plusieurs autres individus sans que des liens de parenté ne les unissent forcément. Bien que cette définition rappelle quelque peu celle de la «famille élargie », elle s'apparente davantage aux « partenariats » féminins appelés spinster clustering par Olwen Hufton et repérés au XVIII ${ }^{\mathrm{e}}$ siècle à Beauvais, Lyon ou Paris mais aussi dans les villages de Le Velay et de Bessin ${ }^{53}$. Nancy Locklin en a retrouvé dans les villes bretonnes où des mères et filles, des tantes et nièces, des sœurs, toutes veuves ou «filles majeures", vivent ensemble pour échapper à la vie fragile ${ }^{54}$. Toutefois, les cohabitations parmi les populations navigantes s'en différencient : l'absence impose à la femme la charge et l'entretien du foyer quand son conjoint est en mer. Lorsque la vie conjugale est marquée par des absences plus ou moins longues et fréquentes, c'est avec son ou sa cohabitant(e) que la femme passe la majeure partie de son temps au quotidien et pas avec son époux. La cohabitation se fait avec une personne seule qui souvent, fait partie de la famille. Il s'agit d'un rapprochement de solitudes féminines, l'une temporaire et l'autre prolongée, pour la plupart : les deux-tiers des cohabitantes vivent avec une autre femme, veuve ou fille majeure, leur mère, une sœur ou une tante. Elle se présente comme une évidence pour certains ménages dès le début de la vie conjugale : Mathieu Gleyo vit avec sa femme et sa belle-mère qui a toujours demeuré avec eux depuis leur

51 Ibid., 9B328, procès-verbal de descente du 11 septembre 1772.

52 Ibid., 4E1508, afféagement du 29 juillet 1768.

53 HufTON O., « Women without men : widows and spinsters in Britain and France in the eighteenth century », Journal of Family History, décembre 1984, n 9, p. 355-376.

54 LocKlin N., Women's work and identity in eighteenth-century Brittany, Chipenham, Ashgate Publishing, 2007, 162 p. 
mariage ${ }^{55}$. L'absence répétée du conjoint peut tout autant y contribuer. Cela commence par quelques nuits passées ailleurs ${ }^{56}$ comme l'avoue Françoise Lemonnier dont beaucoup d'effets se trouvent chez sa mère « vu qu'elle y couche plus souvent que chez elle $^{57} »$. Ces nuits se transforment en un emménagement définitif : Marie Dufée finit par «se retirer» chez sa mère, son mari s'étant embarqué quatre ans auparavant sans avoir donné aucune nouvelle ${ }^{58}$. La dégradation de l'état de santé ou le grand âge entraînent parfois une situation semblable : François Lefeuvre, un navigant de Cancale dont l'épouse est « infirme et grabataire », s'est vu dans l'obligation de s'installer chez sa propre sœur, une veuve, afin qu'elle s'en occupe pendant ses absences sur mer ${ }^{59}$. Si la cohabitation constitue une alternative à la solitude, elle est aussi une réponse à l'irrégularité des salaires maritimes grâce au partage des revenus et des dépenses entre les membres du foyer. C'est ce que sous-entend la femme de Louis Félix Norois en déclarant « demeurer ensemble et en communauté » avec sa tante à Roscoff ${ }^{60}$. L'attitude de Bertranne Portier après la mort en mer de son mari, est plus explicite : vivant avec sa sœur, Vincente Portier, dans une maison de Saint-Ideuc, elle précise au greffier qu'elles ont filé toutes les deux «quatre paquets de filasse ». Elle ajoute que sa sœur «a aidé à gagner les espèces et meubles ci devant estimés pourquoi elle croit en justice qu'il lui revient pour ses bons services savoir l'armoire à quatre battants, le coffre de bois de chêne, et une des vaches $^{61}$ » soit près d'un tiers du montant de l'inventaire. Il s'agit bien d'une reconnaissance du travail effectué dans le ménage, approuvée par les parents présents. Les effets recensés suggèrent que c'est Vincente Portier qui est venue d'installer chez sa sœur et son beau-frère et non l'inverse. Dans les deux cas, la cohabitante vit souvent avec peu d'affaires, quelques hardes, un peu de literie voire des meubles. Elle dispose parfois d'une pièce dans le logement. Malgré ces biens matériels en général réduits, le cohabitant, selon ses moyens, contribue aux dépenses et peut investir dans la communauté.

\section{Conclusion}

Faire le choix de l'horizon maritime a de réelles implications sur la vie de couple et la répartition des tâches et du travail au sein du ménage. Pour l'homme, car dans la majorité des cas, cette orientation vers le large s'est faite sans réelle vocation et plutôt en raison d'un manque de perspectives d'avenir à terre. Pour la femme de marin, car elle doit apprendre à organiser sa vie et celle de son foyer avec ou sans homme à la maison.

55 ADCA, B556, inventaire après décès du 19 mai 1789.

56 Voir GUICHARD-ClAUdiC Y., op. cit., p. 205.

57 ADIV, 4B1027, inventaire après décès du 10 avril 1732.

58 Ibid., 4B5321, inventaire après décès du 5 mai 1789.

59 Ibid., 4E1504, déclaration du 14 mars 1761.

$60 \mathrm{ADF}, 23 \mathrm{~B} 305$, inventaire après décès du 23 octobre 1747.

61 ADIV, 4B3443, inventaire après décès du 20 février 1739. 
Ces ménages de gens de mer frappent par leur pragmatisme et leur inventivité. Ils déploient de véritables stratégies de (sur)vie, souvent combinées les unes avec les autres pour faire face à la précarité de l'existence et assurer l'équilibre économique de la famille, pendant les périodes d'absence du mari. Cette capacité à se débrouiller donne donc aux femmes de marins davantage d'autonomie en bouleversant les relations de pouvoir dans le couple à travers la redistribution des tâches et du travail familial, en faveur des femmes. Cette autonomie reste cependant subordonnée aux besoins du ménage. Ainsi, les associations informelles de femmes, souvent à caractère familial, peuvent être interprétées comme une réponse aux faiblesses de la famille nucléaire, fragilisée par l'absence d'un des conjoints.

Dans ces conditions, le retour des hommes s'annonce difficile : les retrouvailles peuvent vite dégénérer en une situation de conflit car il est difficile de «trouver la bonne distance conjugale ${ }^{62}$ ». Les femmes doivent aussi concilier deux temps dans leur vie : celui de la séparation associé à une grande autonomie et celui de la vie de couple, "normale », qui les renvoie à un statut de femme sous la tutelle de son époux. Néanmoins, cette plus grande liberté, même temporaire et incomplète elles restent soumises au regard de la communauté et aux normes sociales - leur confère un statut intermédiaire, entre la femme mariée à un terrien sédentaire, placée constamment sous son autorité, et la femme un peu plus indépendante mais seule, célibataire ou veuve, qu'elles risquent de devenir. C'est à ce dur apprentissage que fut confrontée Marie-Jacquette Pignot : malgré ses prières, elle ne revit jamais son mari, qui trouva la mort à Callao le 11 avril 1748, pris de « fièvres et de cours de ventre avec flux de sang ${ }^{63} »$. Quant à ses fils, devenus marins, ils disparurent aux Indes, l'un en 1754 et l'autre 4 années plus tard. Marie-Jacquette mourut seule, en 1785 : elle avait alors 75 ans ${ }^{64}$.

62 GUICHARD-ClAUdiC Y, op. cit., p. 20.

63 Henwood P., op. cit., p. 322.

64 Ibid., p.324. 